\title{
Lotfi ABOUDA
}

Université Stendhal - Grenoble 3

\section{Négation, Interrogation et Alternance Indicatif - Subjonctif ${ }^{1}$}

\section{Introduction}

Cet article se propose d'étudier l'alternance modale en français entre l'indicatif et le subjonctif dans les complétives dépendant d'un verbe dit d'opinion (penser, croire, conclure, etc.), employé négativement ou interrogativement :

(1) a Il croit que Pierre est revenu / * soit revenu.

b Croit-il que Pierre est / soit revenu ?

c Il ne croit pas que Pierre est / soit revenu.

Ces types de complétives demeurent parmi les rares structures enchâssées qui permettent l'alternance modale. La tendance générale en français se traduit en effet par l'apparition, dans une complétive donnée, d'un seul mode personnel, à l'exclusion de l'autre :

(2) Il veut

Il ne veut pas que Pierre revienne / * que Pierre reviendra

Veut-il

(3) Il sait

Il ne sait pas que Pierre est revenu / * que Pierre revienne

Sait-il

Les exemples (1)-(3) montrent que la sélection modale dans les complétives entretient un rapport déterminant avec un élément supérieur - en l'occurrence les verbes ${ }^{2}$ croire, vouloir, savoir qui gouverne la phrase enchâssée elle-même.

\footnotetext{
${ }^{1}$ Une version antérieure, et, sur un certain nombre de points, totalement différente, a été présentée au 2e colloque Chronos (Bruxelles, janvier 1997), et publiée sous le titre “ De la variation modale dans les complétives ” dans Le Langage et l'Homme, vol. XXXIII, $\mathrm{n}^{\circ} 1$ (mars 1998).

${ }^{2}$ Par souci de simplification, nous nous contentons de prendre ici le cas où la complétive est dominée par un Verbe. Mais la catégorie syntaxique de l'élément supérieur qui domine la complétive n'a aucune incidence sur la sélection modale. Celle-ci obéit grosso modo aux mêmes mécanismes lexico-syntaxiques, sans égard aux catégories :

(i) La volonté de Pierre que Paul revienne / * revient.

(ii) La constatation que Paul est revenu / * soit reven

Il n'est pas certain que Pierre reviendra / revienne.

Est-il certain que Pierre reviendra / revienne ?

Pour plus de détails, voir H. Huot (1986) \& (1988) et L. Lalaire (1995).
} 
La marque modale se distingue ainsi nettement des autres marques flexionnelles, notamment temporelles et aspectuelles, qui, elles, se déterminent exclusivement à l'intérieur du domaine IP, sans que des éléments extérieurs puissent les influencer d'une manière décisive :

(4) Il m'a dit que Pierre

vient / est venu / venait / était venu / viendrait / serait venu...

Ces données suggèrent l'hypothèse d'appréhender la sélection modale dans les complétives comme le résultat d'un phénomène d'accord entre deux paquets de traits, qu'il nous reste à préciser, dont l'un serait porté par l'élément qui gouverne la complétive, et l'autre serait projeté par la tête fonctionnelle de la complétive.

Après un rappel sommaire du cadre théorique qui va nous servir ici de référence (section 1), nous présenterons (section 2) nos hypothèses générales sur le phénomène de la sélection modale. L'apparition d'un mode donné dans une complétive sera considérée comme un phénomène d'accord entre, d'une part, des traits lexicaux imposés par la catégorie qui gouverne la complétive et, d'autre part, des traits projetés par les têtes fonctionnelles (C et I) de la phrase enchâssée. Ce sera pour nous l'occasion de répondre à trois questions cruciales: (i) le mécanisme d'assignation des traits aux différentes catégories pertinentes (2.1) ; (ii) le mécanisme d'accord entre les différents traits (2.2), et, enfin (iii) la nature des traits en question (2.3). Une fois notre modèle présenté ainsi dans ses grandes lignes, nous nous proposerons (section 3) le traitement de l'alternance indicatif - subjonctif dans les complétives dépendantes d'un verbe d'opinion.

\section{Le cadre syntaxique}

Pour rendre compte du phénomène de la sélection modale - d'abord, dans son ensemble, et, plus particulièrement, dans les complétives dépendantes d'un verbe dit d'opinion -, nous choisissons ici le cadre théorique du Gouvernement \& du Liage (désormais GB) ${ }^{3}$.

Le modèle GB étant aujourd'hui largement familier, nous nous contenterons ici de fournir la structure arborescente - simplifiée - sous (5), qui représente une complétive dépendante d'un verbe $(\mathrm{V} 1)^{4}$, et où les éléments marqués en gras sont soupçonnés de jouer un rôle dans la détermination du mode de la phrase enchâssée :

\footnotetext{
${ }^{3}$ Chomsky (1981), (1982) et (1986). Voir également, pour une présentation relativement exhaustive, Haegeman (1994).

${ }^{4}$ Dans notre terminologie, V1 représente le verbe de la proposition enchâssante, ce qu'on appelle quelquefois le verbe opérateur.
} 
(5)


Cette représentation, qui va servir de référence dans tout le traitement qui suit, n'appelle pas de commentaires particuliers, si ce n'est sans doute de rappeler que, à la fois pour des raisons empiriques et internes à la théorie, on a été amené à admettre l'existence dans CP d'une position pré-IP, autre que COMP - ou C -, à savoir la position Spec C'. COMP accueille principalement les complémenteurs alors que Spec C' accueille les mots qu-, c'est-à-dire, dans le contexte syntaxique précis qui nous occupe ici, les éléments interrogatifs : quand, où, qui, que, etc.

Ajoutons, d'autre part, sans entrer ici dans les détails de la composition interne de I, que notre présentation s'écarte de l'analyse défendue par Pollock (1989) et Chomsky (1991) puisqu'elle considère le Mode comme l'un des constituants de la structure arborescente et hiérarchique de I, et que, de ce fait, la forme insérée sous $\mathrm{V}$ n'est autre que le radical verbal qui prend ses suffixes flexionnels en montant successivement dans les différents noeuds de $\mathrm{I}^{5}$.

\footnotetext{
${ }^{5}$ Plusieurs arguments sont avancés dans Abouda (1997), notamment les chapitres II et III, en faveur de cette analyse qui vise à réadapter la Split INFL Hypothesis aux données flexionnelles du français, qui se caractérise par une morphologie modale assez riche.
} 


\section{Le mécanisme de sélection modale}

La plupart des travaux classiques consacrés aux modes verbaux instaurent une hiérarchie entre l'indicatif et le subjonctif : le premier serait neutre, ne véhiculant aucune valeur sémantique propre, et ne méritant le statut de Mode que "par courtoisie", selon l'expression de Lyons (1970 : 235), par opposition au subjonctif, qui serait le Mode par excellence, véhiculant des vraies valeurs sémantiques.

Cette hiérarchie, qui se révèle tout particulièrement inapte à rendre compte de la variation modale dans un certain nombre de contextes syntaxiques, sera considérée par nous, suite, entre autres, à Bally (1965) et Bonnard ( 1974), comme abusive, et sera abandonnée ${ }^{6}$. L'opposition entre le subjonctif et l'indicatif sera donc traitée ici, non pas en termes d'opposition entre tout et rien, mais plutôt en termes d'opposition entre valeurs sémantiques distinctes mais qui appartiennent à un même plan.

D'autre part, la plupart des travaux qui traitent du subjonctif s'attachent à discriminer et à nommer les différentes "valeurs" de ce mode, négligeant ainsi souvent toute approche monsémiste qui chercherait à dégager le signifié global du subjonctif.

Outre les critiques théoriques générales que l'on peut émettre à l'égard de ces approches strictement polysémistes $^{7}$, nous nous joignons à Lalaire pour qui, dès qu'il s'agit de chercher à déterminer les différentes valeurs du subjonctif, "le risque est grand de n'en avoir jamais fini à les dénombrer" (1995: 1$)^{8}$.

En bref, ainsi que l'écrit en substance Curat (1991), si on obtient le même mode à la suite des idées contradictoires de regret et de désir par exemple, c'est que l'opposition entre regret et désir n'est pas pertinente pour l'explication de l'apparition du mode considéré. Autrement dit, il serait préférable de suivre une piste monosémiste, et de chercher à trouver ce qui est commun à tous les emplois considérés.

De toutes les pistes monosémistes, celle qui a été tracée par Damourette et Pichon nous paraît la plus prometteuse, même s'il sera nécessaire de redéfinir les notions relatives à l'assertion, auxquelles ils ont fait appel pour le traitement des modes verbaux. Nous y reviendrons (§ 2.3. infra).

Enfin, la majorité des travaux classiques qui traitent des modes verbaux se situent exclusivement dans une perspective logico-sémantique. Or, loin du débat classique qui avait opposé, à propos du subjonctif, les tenants des thèses dualistes aux tenants des thèses unitaires, un certain

\footnotetext{
${ }^{6}$ Pour une discussion détaillée, voir Abouda (1997 : 109 sqq.).

${ }^{7}$ Voir sur ce point Confais (1990).

${ }^{8}$ C'est que, comme l'écrivait déjà Moignet (1959 : 7), " si l'on aborde la question des valeurs, on rencontre une confusion bien plus grande encore. On a pu parler de subjonctif désidératif, de jussif, d'injonctif, de prohibitif, de volitif ou volontatif, d'optatif, de debutatif, de concessif, de suppositif, de potentiel, d'aperceptif. [...] Mais il apparaît qu'on n'a guère progressé dans la connaissance de ce mode-Portée quand on a épinglé sur chaque cas d'emploi une étiquette en -tif."

Ajoutons de notre part que ces valeurs sémantiques ne peuvent en aucun cas être considérées comme définitoires du mode subjonctif puisque, d'une part, chacune d'entre elles peut être rendue par un autre mode que le subjonctif, et, d'autre part, ces listes sémantiques, malgré leur longueur, sont loin d'être exhaustives.
} 
nombre de travaux récents, sans négliger l'aspect sémantique de la question, ont souligné l'importance de la syntaxe dans le phénomène de la sélection modale 9 .

Nous verrons ci-dessous que même dans le cas que l'on peut juger le moins syntaxique, comme celui qui nous occupe ici où il y a variation modale, l'aspect syntaxique se révèle décisif dans l'explication de l'apparition de l'un ou l'autre des deux modes considérés. En effet, d'une part, les traits sémantiques responsables de la sélection modale doivent obligatoirement être assignés à des têtes syntaxiques (§ 2.1), et, d'autre part, l'héritage des traits en question doit obligatoirement suivre des chemins syntaxiques précis, i.e. des chemins qui relèvent de la complémentation (§ 2.2).

\subsection{Assignation des traits aux catégories-têtes}

Nous considérons, suivant en cela de nombreux linguistes, que, dans le cas le plus général, le mode de la complétive dépend d'une manière cruciale du terme qui la gouverne, c'est-à-dire en l'occurrence le verbe opérateur, noté V1 dans la configuration (5).

Plus précisément, le mode de la complétive est considéré comme étant déterminé, selon un mécanisme qui sera précisé infra (§ 2.2), par un sème - ou trait sémantique - porté par V1. Ce trait est conçu comme une unité sémantique minimale, non susceptible de réalisation indépendante, et assez abstrait, pouvant ainsi constituer $a$ priori le plus petit dénominateur commun à un nombre importants d'unités lexicales.

La question de la valeur exacte de ce trait sera posée ci-dessous ( $\S 2.3$ ). Nous nous contenterons ici de nous poser la question - théorique - de l'assignation de ce trait - que nous appelons $\mathrm{T}$ - au verbe opérateur V1.

A l'image de ce que l'on fait aujourd'hui dans les grammaires d'unification - et notamment dans le cadre de la Grammaire Syntagmatique Guidée par les Têtes $(H P S G)^{10}$ - nous posons que chaque terme, dès le niveau de son entrée lexicale, doit être associée à une structure de traits prédéfinie, dans laquelle on spécifie ses propriétés phonologiques, syntaxiques et sémantiques.

Dans la sous-structure syntaxique, on spécifie notamment la catégorie grammaticale du terme en question, ainsi que la liste de sa sous-catégorisation (le nombre de ses arguments et leurs catégories grammaticales). Lorsque l'unité lexicale peut admettre pour complément un $\mathrm{CP}$, nous proposerons d'ajouter dans sa structure sémantique le trait $\mathrm{T}$, auquel on assigne une valeur donnée ${ }^{11}$.

\footnotetext{
${ }^{9}$ Voir notamment Abouda (1997), (1998), (1999) et Lalaire (1995).

${ }^{10}$ Pour une introduction à ces modèles, voir par exemple Abeillé (1993).

${ }^{11}$ L'usage de structures de traits prédéfinies permet une telle économie : seules les unités lexicales ayant dans leur liste de sous-catégorisation un complément de type phrastique seront spécifiées, dans leur structure sémantique, pour le trait T.
} 


\section{$2.2 \quad$ Relations syntaxiques}

Deux types fondamentaux de relations syntaxiques seront ici appelés à jouer un rôle déterminant dans l'explication de la variation modale.

La première relation est une relation de type sélectionnel qui existe entre toute tête - qui peut être aussi bien lexicale que fonctionnelle - et son éventuel complément.

Dans la représentation arborescente (5), la complétive - CP - joue le rôle de complément par rapport à V1 qui, lui, en tant que tête, sélectionne le type de CP qu'il est susceptible d'admettre. Or, cela revient simplement à dire que V1 exige que le CP qu'il sélectionne porte un certain nombre de traits sémantiques compatibles avec ses propres traits.

Or, parallèlement, ces traits portés par CP sont en fait nécessairement des traits projetés par la tête de CP : on en arrive ainsi à la seconde relation syntaxique considérée ici comme pertinente, à savoir la relation projectionnelle.

Un syntagme CP, et d'une façon plus générale, tout constituant syntaxique, du moment où il constitue une projection maximale et remplit la fonction de complément, ne peut donc être acceptable que s'il y a accord entre les traits sélectionnés par l'élément supérieur - en l'occurrence V1 - et les traits projetés de la tête, en l'occurrence le complémenteur. Autrement dit, une complétive n'est acceptable que s'il y a un accord entre le système sélectionnel et le système projectionnel.

Pour expliquer rapidement ce mécanisme, on peut observer les phrases (6) :

(6) a *Je me demande que Pierre est parti

b *Je me demande partir

où l'on suppose qu'un verbe comme se demander sélectionne un complément de type interrogatif. Or, aucun trait interrogatif n'est projeté de la tête de la phrase enchâssée : il y a donc une contradiction entre le trait interrogatif exigé par l'élément gouverneur et le trait déclaratif projeté par la tête de la subordonnée, d'où l'exclusion des phrases en question.

Le même mécanisme s'applique mutatis mutandis pour la relation entre le complémenteur tête de CP - et IP qui joue par rapport à C le rôle de complément direct. Pour que IP soit acceptable, il faut que les traits projetés par INFL soient compatibles avec les traits exigés par C.

Pour expliquer la variation modale, il ne sera pas ici nécessaire de faire appel à un quelconque autre mécanisme supplémentaire.

Il suffit pour cela d'ajouter dans la structure de traits de tout élément gouvernant une complétive le trait sémantique $\mathrm{T}$, introduit ci-dessus, qui sera considéré comme responsable de la 
sélection modale. Pour des raisons évidentes d'économie, nous considérerons ici qu'il s'agit d'un trait binaire $[ \pm \mathrm{T}]$ dont la valeur $[+\mathrm{T}]$ serait responsable de l'apparition de l'indicatif et la valeur $[-\mathrm{T}]$ responsable de celle du subjonctif ${ }^{12}$.

D'après le mécanisme présenté ci-dessus, le trait en question serait d'abord porté par V1, et enregistré tel quel dès le niveau de l'entrée lexicale du verbe en question. Les verbes admettant une complétive seront ainsi distingués en deux grandes classes, selon qu'ils sont spécifiés [+ T] ou [- T].

En entrant dans une relation syntaxique avec un complément phrastique, V1 sélectionne des phrases compatibles avec ses propres traits, y compris pour le trait $[ \pm T]$.

Or, ainsi que nous l'avons suggéré ci-dessus, pour qu'il y ait acceptabilité, il faut qu'un trait analogue et ayant la même valeur que celle du trait porté par V1 soit projeté par COMP, tête du CP complément. Nous faisons ici l'hypothèse que le trait projeté par COMP serait lui-même un trait sémantique porté par les différents mots pouvant occuper la position COMP. Cela revient à supposer qu'il serait possible de distinguer dès le niveau lexical entre les différents complémenteurs selon qu'ils sont spécifiés [+ T] ou [- T].

Et à son tour, COMP, mais cette fois-ci en tant qu'élément supérieur qui gouverne un IP complément, sélectionne le type de syntagme IP qu'il est susceptible d'admettre, en exigeant de lui qu'il porte des traits (y compris le trait $[ \pm \mathrm{T}]$ ) analogues aux siens.

Là aussi, pour qu'il y ait acceptabilité, il est nécessaire que COMP sélectionne un trait [+ T] ou [- T] qui soit parallèlement projeté par INFL, tête de IP.

Aucun mot lexical ne pouvant occuper la position INFL, le trait $[ \pm T]$ sera considéré comme étant véhiculé par le morphème de Mode dont nous avons dit ci-dessus (§ 1) qu'il constituait l'un des nœuds de $\mathrm{INFL}^{13}$. Autrement dit, le trait, de nature sémantique, $[ \pm \mathrm{T}]$ peut avoir pour support non seulement des unités lexicales (certains verbes opérateurs, certains complémenteurs et mots qu-) mais également des morphèmes, i.e. les morphèmes de modes.

Ce mécanisme nous semble ici suffisant pour tenter d'expliquer la variation subjonctif indicatif dans le contexte syntaxique d'une complétive.

Reste à déterminer le trait $[ \pm \mathrm{T}]$ en question.

\footnotetext{
${ }^{12}$ Nous sommes d'accord avec l'un des lecteurs anonymes pour dire que ce trait binaire, tel quel, n'est sans doute pas en mesure de rendre compte de l'apparition de l'infinitif. Ce trait nous semble pourtant suffisant pour le traitement de l'alternance Indicatif - Subjonctif, qui constitue l'objet de ce travail. Ajoutons que la solution qui consisterait à donner au trait $\mathrm{T}$ des valeurs directement morphologiques (de types Indicatif, Subjonctif, Infinitif) nous paraît totalement $a d$ hoc, et sans réel pouvoir explicatif, sans même parler des cas où un élément peut sélectionner indifféremment (soit directement, soit suite à une opération syntaxique) une complétive à l'indicatif ou au subjonctif.

${ }^{13}$ Plus précisément, pour être totalement acceptable, cette analyse doit supposer que le nœud Mode constitue la tête de INFL. Cette vision, qui s'écarte de la version standard de la Split INFL Hypothesis (voir Pollock (1989) et Chomsky (1991)), n'est pas sans poser des problèmes techniques au modèle qui nous sert ici de référence, mais elle nous paraît la seule analyse capable de rendre compte des données flexionnelles du français. Voir à ce propos Abouda (1997), notamment les chapitre II et III.
} 
Déterminer le trait lexical qui serait responsable de la variation modale dans les complétives n'est pas une tâche aisée. Le risque est trop grand de se voir construire un modèle logico-sémantique sans véritable assise concrète.

Pour essayer de limiter ce risque, il nous a semblé possible d'essayer de déterminer le trait sémantique en question dans un contexte syntactico-lexical analogue, mais plus simple (contenant moins de variables), à savoir dans les CP indépendants.

En effet, d'après le mécanisme mis en place ci-dessus, lorsqu'il est sélectionné par V1, le trait [+ T] ou [- T] doit parallèlement être porté par les complémenteurs et, par extension, par les éléments $q u$ - qui, en vertu du principe connu sous le nom de Spec-Head Agreement ${ }^{14}$ doivent partager les traits pertinents avec les complémenteurs. Or, puisque les complémenteurs et mots qu- constituent une classe fermée, il est en principe plus aisé de déterminer le trait recherché.

Il suffirait donc en principe d'examiner la variation modale dans le contexte d'un CP indépendant pour déterminer le trait sémantique qui oppose les deux classes de complémenteurs et mots qu- selon qu'ils admettent un IP à l'indicatif ou un IP au subjonctif.

Les données pertinentes dans ce contexte sont bien connues, et relativement simples ${ }^{15}$, comme le suggère le paradigme (7)-(10) :

(7)
a Si j'avais su !
b - Resterez-vous?
- Si je reste ! Si je reste !
c S'il est grand !

(9) a Qu'il vienne !

b Que le ciel vous entende!
(8)
a Qui est venu ?
b Quand viendra-t-il ?

(10) a Que n'écrit-il en prose !

b Que ne l'ai-je su!

c Qu'il était mignon!

La première remarque concerne la conjonction que (à ne pas confondre avec son homonyme, l'élément qu- interrogatif) qui se rencontre à la fois dans les phrases (9) et (10). Puisque cette conjonction peut aussi bien se faire suivre d'une phrase à l'indicatif que d'une phrase au subjonctif, cela suggère qu'elle serait neutre par rapport au trait $[ \pm T]$, responsable de l'apparition du Mode verbal. Or, cela qualifie convenablement la conjonction que, élément sémantiquement vide que Milner (1989) assimile à un sas qui n'a de fonction que syntaxique et peut d'ailleurs dans certaines langues, comme l'anglais, ne pas apparaître.

\footnotetext{
${ }^{14}$ Voir, par exemple, Haegeman (1994).

${ }^{15}$ Il convient toutefois de préciser que ces données, loin de refléter tous les cas qui se présentent, ne font que représenter la tendance générale. On n'est en effet jamais à l'abri d'une 'distorsion modale', comme dans le tour qui l'eût cru ?.
} 
Reste à opposer les autres éléments pré-IP par rapport à un trait sémantique assez général susceptible d'expliquer la variation modale dans la phrase enchâssée.

D'après le mécanisme mis en place ci-dessus, les éléments pré-IP doivent en principe s'opposer par le même trait que celui qui oppose le subjonctif à l'indicatif.

Or, l'une des hypothèses classiques les plus fortement ancrées consiste à décrire l'opposition entre ces deux modes en termes relatifs à l'assertion. Il s'agit d'une hypothèse qui répond à une forte intuition, et qui n'a jamais, à notre connaissance, été remise en cause, malgré des désaccords importants d'interprétation et de terminologie.

Cela justifie ici que l'on puisse être tenté d'exploiter cette piste. Quant à l'adoption elle-même de l'hypothèse en question, elle ne peut se justifier que par son adéquation descriptive et explicative, adéquation qui fera l'objet d'une vérification dans les paragraphes ci-dessous.

En examinant les données présentées sous (7)-(10), on peut immédiatement s'apercevoir que les complémenteurs et mots qu- spécifiés [+ T], c'est-à-dire les éléments suivis d'une phrase à l'indicatif, sont, en rapport avec la notion d'assertion, extrêmement variables et peuvent être rangés en deux classes diamétralement opposées :

(i) ou bien ils expriment une idée de non-assertion : c'est le cas de la conjonction si (à la fois dans les hypothétiques et les interrogatives indirectes totales) qui suspend la valeur de vérité de la proposition qui suit et de tous les éléments qu- interrogatifs (que, qui, quand, lequel, où, etc.) ;

(ii) ou bien les éléments qui expriment, au contraire, l'idée d’une assertion intensifiée, ou d'hyperassertion : c’est le cas des éléments qu- exclamatifs : si, comme, combien, que, quel, etc.

Si le concept d'assertion est réellement adéquat pour l'explication de la variation modale, n'est-il pas pour le moins surprenant que l'on soit amené à regrouper dans une même classe des éléments aussi hétérogènes que l'interrogation, l'hypothèse, la déclaration et l'exclamation, sous prétexte qu'ils ont en commun de gouverner un IP à l'indicatif ?

On trouvera chez Anscombre \& Ducrot (1983), Ducrot (1984) et R. Martin (1983 \& 1987) une série d'arguments sur lesquels peut s'appuyer un tel regroupement. Si en effet le rapport entre l'exclamation et l'assertion peut facilement être établi ${ }^{16}$, nous nous rangeons volontiers aux thèses défendues par ces auteurs pour qui, poser une question revient à poser une " assertion préalable ” (Anscombre \& Ducrot, 1983). Cela semble tout particulièrement justifié à propos des questions partielles, les seules qui nous occupent dans ce paragraphe (elles contiennent un mot qu-) ${ }^{17}$. La même chose peut être dite, mutatis mutandis, à propos de l'hypothèse qui, selon Martin (1987 : 25), “présuppose la vérité de p dans quelque monde possible ”.

\footnotetext{
${ }^{16}$ Pour Curat (1990), l'exclamation est une hyperassertion (une assertion intensifiée).

${ }^{17}$ Nous reviendrons ci-dessous sur les questions totales.
} 
Aussi, parallèlement à l'acception restrictive de l'assertion, qui fait d'elle un équivalent extensif de la déclaration - s'opposant ainsi à la question, la négation, l'hypothèse, etc. -, aurions-nous besoin d'une nouvelle notion, plus générale, qui définisse ce domaine qui englobe non seulement l'assertion, mais également toutes les opérations linguistiques qui présupposent une assertion préalable. Nous appellerons ce domaine le domaine intra-assertif que nous définissons comme le domaine où est assignée, soit directement soit indirectement, une valeur de vérité à une proposition p quelconque.

Cette définition, assez abstraite, mérite quelques précisions :

(i) la valeur de vérité en question peut être de différents types : elle peut prendre tout aussi bien la forme de [+ Vrai], [+ Faux], que la forme de $[ \pm$ Vrai $]$ ou $[ \pm \text { Faux }]^{18}$;

(ii) nous ne considérons pas que le seul être linguistique susceptible d'attribuer dans un énoncé une valeur de vérité soit le locuteur : toutes les fois qu'il y a attribution d'une valeur de vérité, même de la part d'un être distinct du locuteur ${ }^{19}$, nous nous situons dans le domaine intra-assertif ;

(iii) la conception que nous défendons n'assimile pas l'attribution d'une valeur de vérité à p à la garantie de la conformité de p avec le monde: ni le mensonge, ni la mauvaise foi, ni encore l'hypothèse ne sont selon nous incompatibles avec le domaine intra-assertif.

Nous donnons ainsi au domaine intra-assertif une acception générale, présupposant simplement l'attribution d'une valeur de vérité, que ce soit dans un Univers, dans un Anti-Univers ou encore dans un Hétéro-Univers, pour employer la terminologie de Martin (1983).

Autrement dit, toutes les fois que nous avons affaire à une assertion ou à une hyperassertion, c'est-à-dire à une attribution directe d'une valeur de vérité à un énoncé $\mathrm{p}$ de la part du locuteur, nous nous situons dans le domaine intra-assertif. Mais quelquefois, même dans le cas d'une question - ceci est particulièrement visible dans le cas des questions partielles - ou dans le cas d'une négation réfutative, nous nous situons tout de même dans le domaine intra-assertif dès lors qu'il y a attribution indirecte - préalable - d'une valeur de vérité à p, objet de la négation ou de la question.

Le domaine intra-assertif est ainsi conçu comme un domaine assez vaste, qui correspond à une échelle allant de l'assertion intensifiée à la négation, en passant par certains types de questions. Il s'oppose au domaine extra-assertif, où aucune valeur de vérité n'est attribuée (dans aucun des modes ni des univers de croyance), et où les notions même de vérité et de fausseté ne sont pas pertinentes. La proposition p se présente alors comme ni Vraie, ni Fausse, comme une notion pure, diraient Damourette \& Pichon. Le domaine extra-assertif, s'opposant d'une part à un pôle franchement positif l'assertion intensifiée - et, d'autre part, à un pôle franchement négatif - la négation -, se trouverait alors réduit au point central de l'échelle bidirectionnelle que constitue le domaine intra-assertif : dès qu'une proposition quitte ce point zéro, elle se voit attribuer une valeur de vérité et pénètre le domaine

\footnotetext{
${ }^{18}$ Voir Martin (1983).

${ }^{19}$ Voir la théorie polyphonique développée notamment par Ducrot (1984).
} 
intra-assertif. Ceci revient à dire qu'une proposition p se situe dans le domaine extra-assertif si elle ouvre un véritable choix polaire. Dès qu'elle obtient une orientation donnée, elle quitte ce domaine.

Si on revient maintenant aux deux groupes, isolés ci-dessus, de complémenteurs et mots ququi gouvernent un IP à l'indicatif, on peut aisément les regrouper au sein d'une même classe, ayant en commun un sème intra-assertif.

Si cette analyse est acceptable, le trait que l'on est amené à donner par défaut aux éventuels éléments pré-IP qui déclenchent l'apparition du subjonctif est un trait qui doit les situer au sein du domaine extra-assertif.

En conclusion de ce paragraphe, nous dirons que les phrases qui se présentent comme des syntagmes CP dont la tête porte un trait intra-assertif (soit directement si la position C est occupée par un élément lexical spécifié positivement pour ce même trait, soit indirectement via la transmission de ce même trait par un élément occupant la position Spec $C^{\prime}$ à sa tête) seront systématiquement à l'indicatif.

Au contraire, les phrases qui se présentent comme des syntagmes CP dont la position-tête porte un trait extra-assertif seront systématiquement au subjonctif.

\section{La variation modale dans les complétives dépendant d'un verbe d'opinion}

Les interrogatives indirectes (partielles ou totales) sont systématiquement à l'indicatif. Ce phénomène peut désormais recevoir l'explication suivante. Une interrogative indirecte reçoit nécessairement une orientation positive - particulièrement visible dans le cas d'une interrogation partielle $-^{20}$, la situant dans le domaine intra-assertif.

En effet, ainsi que l'affirme en substance Confais (1990), une question comme :

(11) Je me demande depuis quand Pierre habite à Paris

présuppose un énoncé déclaratif sous-jacent de type :

(12) Pierre habite à Paris depuis [...].

D'autre part, même dans le cas d'une question totale, plusieurs linguistes (Ducrot, Anscobre, Confais, Martin, etc.) parlent d'une assertion préalable. “ Demander si Pierre est là, écrit Martin (1983 : 263), c'est envisager au moins un état du monde où Pierre serait là. Si j'estime globalement exclu qu'il soit là, je n'ai aucune raison de poser la question. ” 
En fait, la variation modale n'est observée en français moderne que dans le contexte d'une complétive en que. Le mécanisme décelé ci-dessus reste ici opérationnel sans qu'il soit nécessaire de faire appel à une quelconque autre stipulation supplémentaire : puisque la conjonction que, jugée comme neutre par rapport au trait [intra/extra-assertif], peut aussi bien admettre le subjonctif que l'indicatif, c'est le trait qui lui est imposé par l'élément qui la gouverne - en vertu de la relation sélectionnelle - qui serait responsable de la sélection de l'un ou de l'autre des deux modes dans le IP enchâssé.

Cette analyse semble en mesure d'apporter une explication satisfaisante à un nombre de cas observables. Ainsi, par exemple, dans des phrases comme (13) :
a Je crois qu'il est parti
b Il a dit que Pierre était parti

l'on suppose que l'apparition de l'indicatif est due à la transmission du trait intra-assertif, inhérent à des verbes comme croire ou dire, employés positivement.

Parallèlement, l'apparition du subjonctif est expliquée comme le résultat de la transmission d'un trait extra-assertif.

En effet, certains verbes portent d'une manière inhérente un trait extra-assertif qu'ils imposent alors aux CP qu'ils gouvernent. C'est par exemple le cas des verbes soulignés dans les phrases suivantes :
(14) a Je veux qu'il parte
b Je regrette qu'il soit absent

qui ne présentent pas la proposition p (dans l'enchâssée) comme l'objet d'une assertion, mais comme une notion pure, conçue en dehors de toute considération relative à l'assertion ${ }^{21}$.

\footnotetext{
${ }^{20}$ Voir notamment Confais (1990) et Delaveau (1990). type :

(i) Je dis que je viendrai quelqu'un peut directement remettre en cause la vérité de la proposition enchâssée par un simple :

(ii) C'est faux ! Tu ne viendras pas !

le même processus est exclu pour une phrase comme :

(iii) Je regrette que Pierre soit absent

où seul le verbe au principal peut être remis en cause :

(iv) C'est faux ! Tu en es content / *il n'est pas absent.
}

${ }^{21}$ Voir sur ce point Damourette \& Pichon. Il faut bien comprendre ici que si la proposition enchâssée de (14b) est conforme au monde (on ne peut pas regretter quelque chose qui n'a pas eu lieu), ce n'est pas cette conformité qui est soulignée par le locuteur : la proposition enchâssée ne fait pas ici l'objet d'une assertion, mais de l'expression d'un regret. Si à une phrase de 
Quelquefois, les données sont un peu plus difficiles : V1 porte bien, en tant qu'unité lexicale, un trait intra-assertif, sans que le subjonctif soit exclu, comme c'est le cas dans les phrases (15) :

$\begin{array}{lll}\text { (15) } & \text { a } & \text { Dis-lui qu'il vienne ! } \\ & \text { b } & \text { Imagine qu'il ait eu un empêchement ! }\end{array}$

par opposition aux phrases (16) :

(16) a Il m'a dit qu'il est venu.

b J'imagine qu'il a eu un empêchement.

Le phénomène s'explique aisément si on pose que l'injonction déclenche une règle syntaxique qui réécrit le trait sémantique [intra-assertif] affecté à certains verbes gouvernant une complétive en trait [extra-assertif]. Cette règle peut par exemple prendre la forme suivante :

$$
\text { dire }_{\text {[intra-assertif] }} \longrightarrow \text { injonction } \longrightarrow \text { dire }_{[\text {[extra-ssertif] }}
$$

et correspond bien, sémantiquement, à la nuance que l'on peut attacher à chacun des deux emplois considérés. Mais il s'agit d'une règle contrainte qui, pour être appliquée, nécessite un rapport de coréférence entre l'objet indirect du verbe dire et le sujet de la phrase enchâssée...

Le même phénomène se rencontre dans l'exemple suivant :

(18) Si je pensais qu'il fût un bon candidat, ...

par opposition à l'exemple (19) correspondant sans l'expression d'une hypothèse :

(19) Je pense qu'il est un bon candidat.

Là aussi, on peut proposer une règle, optionnelle cette fois-ci, qui réécrit, sous l'effet d'une hypothèse, le trait intra-assertif du verbe penser en trait extra-assertif. Il convient ici de dissiper dès maintenant un malentendu possible: nous ne disons pas que l'emploi du subjonctif est dû à l'expression d'une hypothèse. Nous avons en effet affirmé ci-dessus que l'hypothèse présuppose une assertion préalable, et se situe alors plutôt dans le domaine intra-assertif. Pour expliquer le subjonctif dans la subordonnée de (18), nous disons quelque chose de différent : l'hypothèse, lorsqu'elle est forte, constitue l'une des opérations qui peuvent neutraliser le trait intra-assertif de V1. La force donnée à l'hypothèse est du seul ressort du locuteur, puisqu'une phrase comme (20) : 
est tout à fait acceptable.

Dès lors, on comprend que certains verbes puissent admettre aussi bien le subjonctif que l'indicatif, selon la nuance ou la coloration (plus ou moins assertive ou au contraire hors-assertive) que le locuteur veut lui donner, comme dans les exemples (21) :

$$
\begin{array}{lll}
\text { (21) a } & \text { Je suppose qu'un moine est toujours charitable (La Fontaine) } \\
\text { b } & \text { Je suppose que les hommes soient éternels sur la terre (La Bruyère) }
\end{array}
$$

qui contiennent deux emplois du verbe supposer, auxquels le Petit Robert réserve une seule entrée, et qui ne se distinguent qu'en ce que le premier donne, à titre d'hypothèse, à la proposition subordonnée une valeur assertive alors que le second se contente de la présenter comme une notion pure.

\subsection{Complétives dans la dépendance d'un verbe d'opinion employé négativement}

Les données, épurées, peuvent être présentées comme dans le paradigme (22)-(23) :

(22) a Je pense que Pierre est un bon candidat.

b *Je pense que Pierre soit un bon candidat.

(23) a Je ne pense pas que Pierre est un bon candidat.

b Je ne pense pas que Pierre soit un bon candidat.

Ce paradigme suggère que dès que le verbe supérieur d'opinion est employé négativement, le subjonctif qui était exclu dans la phrase positive correspondante, devient possible, voire très largement majoritaire. Il convient toutefois de préciser que la négation en question est une négation totale avec ne... pas. ${ }^{22}$

L'explication du caractère obligatoire de l'indicatif dans les subordonnées des phrases (22) ne pose, dans le modèle défendu, aucun problème. En effet, en dehors d'un phénomène syntaxique qui viendrait modifier le trait sémantique porté par le verbe penser, celui-ci est spécifié [intra-assertif] et sélectionne tout naturellement un CP à l'indicatif.

Ce sont les phrases (23) qui posent deux types de problèmes :

\footnotetext{
${ }^{22}$ Il s'agit ici naturellement d'une simplification puisque plusieurs autres paramètres (comme le temps du verbe supérieur ou encore la personne) interviennent et rendent l'emploi du subjonctif beaucoup plus difficile, voire quelquefois totalement exclu. Pour une présentation plus exhaustive de ces données, on peut se rapporter à Huot (1986).
} 
(i) par quel mécanisme le verbe penser vient-il à admettre l'indicatif dans la subordonnée?

(ii) comment rendre compte du caractère facultatif du mécanisme en question (puisque le subjonctif n'est pas obligatoire dans un tel contexte)?

A la première question, le traitement défendu ici propose une solution relativement simple. Nous posons que la négation totale constitue l'une des opérations syntaxiques qui peuvent déclencher une règle de réécriture du sème porté par le verbe opérateur. La règle en question peut prendre la forme suivante :

penser [intra-assertif]<smiles>C[14CH2][12CH2]</smiles>

penser [extra-assertif]

En langage moins formel, nous dirons que la négation totale, lorsqu'elle affecte un verbe d'opinion, annule sa force assertive, et le trait intra-assertif que de tels verbes portaient au niveau lexical se voit alors supplanter par le trait extra-assertif. L'apparition du subjonctif dans les subordonnées gouvernées par de tels verbes s'explique ainsi aisément.

C'est la réponse à la seconde question qui s'annonce plus délicate : pourquoi la règle (24) ne s'applique pas toujours?

Pour y répondre, il convient tout d'abord de rappeler que, sur le plan sémantique, on s'accorde généralement à dire que les phrases (23a-b) ne sont pas entièrement synonymes : il semble bien que l'on assiste à une nuance sémantique selon la forme modale employée dans la complétive ${ }^{23}$. Le tour au subjonctif est en effet généralement présenté comme exprimant une réserve plus grande du locuteur vis-à-vis du contenu de la subordonnée dont il ne prend pas en charge la négation tranchée alors que la phrase à l'indicatif, exprimant plutôt une négation plus catégorique, serait paraphrasable par je crois que Jean n'est pas un bon candidat en analogie avec un procédé appelé quelquefois transfert de négation qui consiste à dire je ne dis pas que... pour je dis que... ne... pas.

Plus précisément, une phrase comme :

(25) Je pense que Pierre n'est pas un bon candidat

constitue une paraphrase naturelle pour l'exemple (23a) à l'indicatif, mais non pour l'exemple (23b) au subjonctif, où l'on présente le contenu de la subordonnée d'une manière plus atténuée.

De là à suggérer que les deux négations dans (23a) et dans (23b) n'ont pas la même origine syntaxique il n'y a qu'un pas. Un pas que nous franchissons d'autant plus volontiers que plusieurs

\footnotetext{
${ }^{23}$ Voir, par exemple, Huot (1986) et Curat (1991).
} 
indices, soulignés par Huot (1986), suggèrent clairement que les deux négations n'ont pas la même portée.

D'abord, comme l'écrit Huot (1986 : 94), “ s'il est tout à fait possible de relier par ni deux complétives à l'indicatif ou deux complétives au subjonctif, il semble exclu qu'on puisse coordonner de la même façon, après un verbe d'opinion employé négativement, une complétive à l'indicatif et une au subjonctif ” :

(26) a Je ne pense pas que Pierre est un bon candidat ni qu'il est motivé.

b Je ne pense pas que Pierre soit un bon candidat ni qu'il soit motivé.

c $\quad$ ??*Je ne pense pas que Pierre est un bon candidat ni qu'il soit motivé.

d Je ne pense pas que Pierre soit un bon candidat ni qu'il est motivé.

Cela suggère fortement que la négation n'a pas dans les deux cas la même origine.

Autre indice intéressant nous est fourni par les constructions clivées. Les données suivantes :

a Je ne pense pas que Pierre soit un bon candidat

a' ??Ce que je ne pense pas, c'est que Pierre est un bon candidat

a" $\quad$ Ce que je pense, c'est que Pierre n'est pas un bon candidat

b Je ne pense pas que Pierre soit un bon candidat

b' ??*Ce que je ne pense pas, c'est que Pierre soit un bon candidat

b" $\quad$ *Ce que je pense, c'est que Pierre ne soit pas un bon candidat.

montrent que la construction clivée est exclue avec une subordonnée au subjonctif, mais qu'elle est naturelle avec une subordonnée à l'indicatif, si la négation n'affecte plus le verbe introducteur, mais plutôt le verbe de la subordonnée. Cela suggère fortement, à notre avis, que dans une phrase comme (23a) avec une subordonnée à l'indicatif la négation a pour origine la phrase enchâssée. Or cela est tout à fait plausible puisque les verbes d'opinion font partie des verbes dits à "montée de la négation"24.

Cette analyse semble pouvoir être confirmée par la reprise pronominale de la subordonnée gouvernée par un verbe d'opinion employé négativement : lorsque la subordonnée est à l'indicatif, la présence du pronom de reprise le semble obligatoire ; lorsque la subordonnée est au subjonctif, il semble au contraire que la seule répétition du verbe opérateur (sans le pronom) soit préférable ${ }^{25}$ :

(28) a - Tu ne penses pas que Pierre est un bon candidat ?

- Non, je ne le pense pas

\footnotetext{
${ }^{24}$ Voir sur ce point Delaveau (1990).

${ }^{25}$ Voir également sur ce point Huot (1986).
} 
??* - Non, je ne pense pas

b - Tu ne penses pas que Pierre soit un bon candidat ?

?? - Non, je ne le pense pas

- Non, je ne pense pas.

Cette solution revient donc à admettre pour la négation affectant un verbe d'opinion deux origines distinctes :

(i) elle peut d'abord avoir pour origine la phrase principale : dans ce cas, elle annule le trait intra-assertif porté par le verbe opérateur, en application de la règle (24), et on obtient dans la subordonnée le subjonctif, comme le résultat de la sélection d'un trait extra-assertif ;

(ii) elle peut, au contraire, avoir pour origine la phrase subordonnée, avant de monter jusqu'à la principale : on suppose que cette montée intervient tardivement, ce qui empêche la règle (24) de s'appliquer, et l'indicatif apparaît.

Cette solution rend compte de la nuance sémantique entre la phrase (23a) et son équivalent au subjonctif - nuance décrite, entre autres, par Huot et Curat - et propose un cadre général facilement exploitable pour traiter la variation dans d'autres contextes.

Ainsi, convient-il par exemple de rappeler, suite à R. Martin (1983), que non seulement, comme il est dit ci-dessus, les verbes d'opinion qui, employés positivement, demandent l'indicatif peuvent, sous l'effet d'une négation, admettre le subjonctif, mais en plus, certains verbes épistémiques qui entraînent le subjonctif, peuvent en contexte négatif, se construire avec l'indicatif. On obtient ainsi le cas de figure strictement inverse de celui présenté ci-dessus. C'est, par exemple, le cas du verbe douter dans les phrases (29) :

(29) a Je doute que Pierre soit un bon candidat

b Je ne doute pas que Pierre soit / est un bon candidat

où, en accord avec les hypothèses énoncées ci-dessus, nous pouvons considérer que sous l'effet de la négation totale, le verbe douter voit son trait extra-assertif s'annuler, d'où, selon le même mécanisme que celui décrit ci-dessus, l'emploi de l'indicatif dans la subordonnée. Il reste que s'il nous semble clair qu'il y a une nuance sémantique en rapport avec le trait intra/extra-assertif selon que la subordonnée est au subjonctif ou à l'indicatif ${ }^{26}$, l'explication du caractère facultatif de l'effet de la négation nous paraît en l'occurrence plus délicate ${ }^{27}$.

\footnotetext{
${ }^{26}$ Lorsqu'il gouverne une complétive à l'indicatif, le verbe douter employé négativement a un sens proche de croire, voire être sûr.
} 


\subsection{Complétives dans la dépendance d'un verbe d'opinion employé interrogativement}

Les données pertinentes dans ce cas sont analogues mutatis mutandis à celles discutées dans le paragraphe ci-dessus. Pour les résumer rapidement, nous pouvons nous appuyer sur la description de Huot (1986) qui démontre clairement que le subjonctif n'est vraiment admis (et courant) que si le verbe d'opinion se trouve dans une construction interrogative totale caractérisée par l'inversion du clitique sujet :

(30) Croit-il que Jean est / soit un bon candidat?

C'est que ni l'interrogation partielle, ni l'interrogation totale intonative, ni celle en est-ce que, n'admettent (ou n'admettent naturellement) le mode subjonctif dans la subordonnée gouvernée par le verbe d'opinion. C'est ce qu'on peut déduire du paradigme (31) (qui est, là aussi, loin de rendre compte de toutes les restrictions signalées dans les différents travaux) :

(31) a Qui croit que Jean est / ??soit un bon candidat ?

b Depuis quand Pierre croit-il que Jean est / *soit un bon candidat ?

C $\quad$ Il croit que Jean est / *soit un bon candidat ?

d $\quad$ Est-ce qu'il croit que Jean est / *soit un bon candidat ?

Une piste à écarter d'emblée consisterait à exclusivement lier l'apparition du subjonctif dans la complétive au phénomène syntaxique de l'inversion du clitique sujet, sans tenir compte de l'interrogation. Les données sont sur ce point parfaitement claires : ce n'est pas l'inversion, en tant que telle, qui déclenche l'apparition du subjonctif :

(32) a Aussi croit-il qu'il est / * soit malade.

b $\quad$ Sans doute croyait-il qu'il était / * fût malade.

Pour expliquer l'apparition du subjonctif dans (30), l'hypothèse globale que nous défendrons est analogue, mutatis mutandis, à celle avancée dans la section précédente pour expliquer l'apparition de ce mode suite à un verbe d'opinion affecté par une négation totale. Elle consiste à considérer l'interrogation totale avec inversion du clitique sujet (en abrégé I.T.I.V.) comme l'un des facteurs qui peuvent déclencher dans certains contextes, qu'il nous reste à expliciter, une règle réécrivant le trait intra-assertif porté initialement par le verbe d'opinion en un trait extra-assertif :

Verbe d'opinion $_{\text {[intra-assertif] }}$

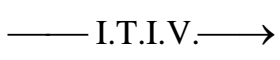

Verbe d'opinion ${ }_{\text {[extra-assertif] }}$

\footnotetext{
${ }^{27}$ Dans la section 3.2. on trouvera une analyse du caractère facultatif du subjonctif dans la dépendance d'un verbe d'opinion
} 
Reste à justifier ce traitement et à répondre à deux questions cruciales :

(i) qu'est-ce qui distingue l'interrogation totale avec inversion du clitique sujet de tous les autres types d'interrogations, permettant ainsi, dans certains cas, au verbe d'opinion de gouverner une complétive au subjonctif ?

(ii) comment expliquer le caractère facultatif de la règle (33) ou - ce qui revient au même pourquoi le subjonctif n'est-il pas, dans le contexte en question, obligatoire?

Pour répondre à la première question, il suffit d'admettre ici que la raison pour laquelle ni l'interrogation partielle, ni l'interrogation intonative ni celle en est-ce que, ne déclenchent une règle de type (33) est qu'elles supposent une assertion préalable, ce qui est suffisant à les maintenir dans le domaine intra-assertif.

En effet, à propos de l'interrogation partielle, nous avons déjà avancé ci-dessus ce traitement, défendu par plusieurs linguistiques dans des cadres logico-sémantiques différents.

La même analyse peut, nous semble-t-il, être avancée à propos des deux autres types de l'interrogation totale. Ceci nous paraît tout particulièrement naturel à propos de l'interrogation intonative qui conserve l'ordre des mots de la phrase assertive correspondante : ce type de tour, loin de constituer une véritable question qui ouvrirait un choix polaire, s'apparente plutôt à une demande de confirmation. Delaveau (1990) cite cet exemple de Proust qui illustre bien notre propos :

"Il habite à Balbec ?" chantonna le baron d'un air si peu questionneur qu'il est fâcheux que la langue française ne possède pas un signe autre que le point d'interrogation pour terminer ces phrases apparemment si peu interrogatives. ${ }^{28}$

Les interrogatives en est-ce que conservent elles aussi l'ordre des mots de la phrase déclarative correspondante. Aussi, même si elles sont plus 'interrogatives' que les interrogations intonatives, elles seront considérées comme véhiculant une assertion préalable, qui donne à la phrase une orientation plutôt positive ou négative. Des trois questions suivantes :

$\begin{array}{ll}\text { (35) a } & \text { Tu viens avec nous ? } \\ \text { b } & \text { Est-ce que tu viens avec nous ? } \\ \text { c } & \text { Viens-tu avec nous ? }\end{array}$

seule la dernière semble véhiculer une véritable question, objective, et sans la moindre orientation assertive. Parmi les indices dont on dispose à l'appui de cette analyse, on peut indiquer ici que des deux phrases suivantes :

employé interrogativement, qui pourrait constituer une piste intéressante pour l'explication du phénomène traité ici.

${ }^{28}$ Proust, Sodome et Gomorrhe, Pléiade, t. 2. 
$\begin{array}{ll}\text { (36) a } & \text { Pourriez-vous m'accompagner ? } \\ \text { b } & \text { Est-ce que vous pourriez m'accompagner? }\end{array}$

seule la première semble exprimer une véritable demande polie : le tour en est-ce que s'accompagne d'une demande plus ferme, plus insistante, orientée.

En bref, nous pouvons considérer ici que seule l'interrogation totale avec inversion du clitique sujet ouvre un véritable choix polaire, sans la moindre orientation assertive. Elle se présente comme la forme interrogative la plus neutre dans le sens où elle ne permet pas de donner au contenu questionné une direction déterminée, vers une valeur positive ou une valeur négative. Ce n'est pas le cas des autres types d'interrogation, qui restent, d'une manière plus ou moins claire, dans le domaine intra-assertif, parce qu'ils réfèrent à des tours assertifs sous-jacents. C'est pour cette raison, selon nous, que seule l'interrogation totale est susceptible de déclencher la règle (33). D'où l'apparition du subjonctif dans une complétive gouvernée par un verbe d'opinion affecté par ce type de question.

Demeure la question, posée ci-dessus, concernant le caractère facultatif de la règle (33) : pourquoi le subjonctif n'est-il pas obligatoire dans un tel contexte ?

Une réponse qui nous semble possible, mais qui devrait être examinée de plus près dans un autre cadre, proviendrait de la nuance sémantique que nous croyons déceler entre les deux tours au subjonctif et à l'indicatif. En examinant le couple suivant de phrases :

(37) a Crois-tu que Pierre soit malade ?

b Crois-tu que Pierre est malade ?

nous pouvons en effet constater que le tour avec le subjonctif est plus 'objectif' dans la mesure où il ne véhicule aucune orientation assertive, ni dans la principale ni dans la subordonnée. Tel n'est pas le cas, nous semble-t-il, du tour équivalent avec l'indicatif qui véhicule, dans la subordonnée, un aspect assertif. Une explication possible, mais qui demande à être confirmée, serait de dire que l'assertion que véhicule l'indicatif provient de l'opinion du locuteur ${ }^{29}$ qui demanderait simplement à l'interlocuteur s'il croit ou non comme lui à la vérité de la proposition questionnée. Peut-être trouverait-on une confirmation à cette analyse dans la différence d'acceptabilité que nous croyons déceler entre les deux phrases suivantes :

\footnotetext{
${ }^{29}$ Si elle est confirmée, cette analyse peut recevoir un traitement convenable dans le cadre de la théorie polyphonique de Ducrot.
} 

??Crois-tu, comme moi, que Pierre soit malade?

b Crois-tu, comme moi, que Pierre est malade ?

Ce traitement revient de fait à considérer la règle (33) comme obligatoire, s'appliquant à chaque fois dans le contexte approprié. L'apparition de l'indicatif dans la phrase (37), cas considéré en l'occurrence comme marqué, serait alors le résultat d'une seconde règle, s'appliquant dans les frontières du IP, qui remplace le subjonctif par l'indicatif.

Cette solution permet, nous semble-t-il, de proposer des traitements plausibles pour un certain nombre de phénomènes difficiles à expliquer, sans qu'il soit nécessaire de faire appel à des mécanismes supplémentaires . Ainsi en est-il du subjonctif qui peut apparaître dans la complétive profondément enchâssée d'une construction parenthétique, lorsque V1 est lui-même employé interrogativement (avec inversion du clitique sujet), comme dans (39) :

(39) Crois-tu que Pierre pense que Jean soit un bon candidat ?

Il en est de même pour une restriction, signalée par Huot, qui concerne l'impossibilité du subjonctif dans la complétive dépendante d'une construction interro-négative (avec inversion du clitique sujet), comme dans (40) :

(40) Ne pense-t-il pas que Jean est / *soit un bon candidat?

où l'on peut supposer que l'effet de l'interrogation et celui de la négation s'annulent mutuellement, bloquant ainsi les deux règles susceptibles de remplacer le trait intra-assertif porté le verbe d'opinion par un trait extra-assertif. Sur le plan sémantique, cette solution rend bien compte d'une phrase comme (40) qui véhicule un sens très proche de la phrase assertive correspondante (Il pense que Jean est un bon candidat).

\section{En guise de conclusion}

Pour des raisons évidentes d'économie, cette étude ne s'est arrêtée que sur les cas jugés les plus saillants, en évitant certaines questions et en traitant certaines autres d'une manière quelque peu elliptique. Conscient de l'impossibilité d'offrir ici un traitement exhaustif à une question aussi complexe et controversée, nous avons essayé ainsi de privilégier la présentation générale du modèle proposé.

Celui-ci, on a pu le constater, ne s'écarte pas beaucoup des descriptions classiques, notamment celles de Damourette \& Pichon, qu'il juge globalement adéquates, et sur lesquelles il s'appuie. 
Il s'en distingue pourtant sur un certain nombre de points, dont nous rappellerons ici les plus importants :

d'abord, ce traitement réhabilite la syntaxe dans un domaine qui était du ressort exclusif de la sémantique. En effet, d'une part, le phénomène de la variation modale est ici expliqué par des traits sémantiques qui sont obligatoirement assignés à des catégories-têtes (les verbes opérateurs, les complémenteurs, le nœud 'mode' considéré comme la tête de INFL), et, d'autre part, les relations sélectionnelles qui assurent la prorogation des traits sémantiques sont obligatoirement des relations de complémentation ;

ensuite, il nous a semblé nécessaire de redéfinir les notions sémantiques cruciales relatives à l'assertion, en instaurant un nouveau concept qui rende compte des différents liens, soulignés par plusieurs linguistes, entre l'assertion, certaines négations, certaines interrogations et l'hypothèse : c'est le domaine intra-assertif défini comme le domaine où une valeur de vérité est attribuée, même d'une manière sous-jacente ;

nous avons enfin mis en place des règles transformationnelles qui sont déclenchées par des phénomènes syntaxiques, telles que la négation totale ou l'interrogation directe totale, et qui réécrivent le trait intra-assertif en trait extra-assertif, et vice versa.

Le traitement de la variation modale est ainsi assuré par des procédures explicites et falsifiables, tenant compte aussi bien de l'aspect proprement syntaxique que des différentes nuances sémantiques que l'on peut attacher à l'un ou à l'autre des deux modes considérés.

Les procédures mises en places, selon un même mécanisme global, assurent au modèle en question une généralité qui pourrait lui permettre de traiter la variation modale dans d'autres contextes.

\section{REFERENCES}

ABEILLE, A. (1993). Les Nouvelles syntaxes. Grammaire d'Unification et analyse du français, Paris : Armand Colin.

ABOUDA, L. (1997). Syntaxe et sémantique du conditionnel en français moderne, Thèse, Université Paris 7.

ABOUDA, L. (1998). “ Vers une localisation syntaxique des modes verbaux ”, Cahiers Chronos, 3, 293-322, Amsterdam : Rodopi.

ABOUDA, L. (1999). “ Les modes verbaux dans les phrases indépendantes ”, Travaux de linguistique, 38, 89-108, Louvain.

ANSCOMBRE, J.-Cl. \& DUCROT, O. (1983). L'argumentation dans la langue. Bruxelles, P. Mardaga. 
BALLY, Ch. (1965). Linguistique générale et linguistique française. 4e édition revue et corrigée. Berne, Francke.

BONNARD, H. (1974). Les Axiomes temps et mode. Le Français moderne, 42, 1, 72-89.

CHOMSKY, N. (1981). tr.fr. (1991). La Théorie du Gouvernement et du Liage, Paris : Seuil.

CHOMSKY, N. (1982). tr.fr. (1987). La nouvelle syntaxe, Paris : Seuil.

CHOMSKY, N. (1986). Barriers, Cambridge, Mass. : MIT Press.

CHOMSKY, N. (1991). “ Some Notes on Economy of Derivation and Representation ”, in R. Freidin (ed), Principles and Parameters in Comparative Grammar, Cambridge, Mass. : MIT Press.

CONFAIS, J.-P. (1990). Temps, Mode, Aspect. Toulouse, Presses Universitaires de Mirail.

CURAT, H. (1991). Morphologie verbale et référence temporelle en français moderne, Genève : Droz.

DAMOURETTE, J. \& PICHON, E. (1911-1927). Des Mots à la pensée, t. V, éd. d'Artrey, 1970

DELAVEAU, A. (1990). La Conjonction "si" dans ses emplois interrogatif et conditionnel en français moderne. Thèse de doctorat d'Etat, Université de Paris-7.

DUCROT, O. (1984). Le Dire et le dit, Paris : Minuit.

HAEGEMAN, L. (1994). Introduction to Government and Binding Theory, Oxford : Blackwell.

HUOT, H. (1986). “ Le Subjonctif dans les complétives : subjectivité et modalisation ”, in M. Ronat \& D. Couquaux, La Grammaire modulaire, Paris : Minuit, 81-111.

HUOT, H. (1988). “ Quelques conditions d'apparition du subjonctif : la notion de classifiance et le trait [ $\pm \mathrm{QU}]$ ”, Recherches nouvelles sur le langage. DRL, Paris 7.

LALAIRE, L. (1995). La variation modale dans les subordonnées à temps fini du français moderne. Thèse, Université Paris 7.

LYONS, J. (1970). Linguistique générale. Paris, Larousse.

MARTIN, R. (1983). Pour une logique du sens, Paris : PUF.

MARTIN, R. (1987). Langage et croyance, Bruxelles : Pierre Mardaga, éditeur.

MILNER, J.-C. (1989). Introduction à une science du langage, Paris : Seuil.

MOIGNET, G. (1959). Essai sur le mode subjonctif en latin postclassique et en ancien français, Paris : PUF.

POLLOCK, J.-Y. (1989). “ Verb movement, UG and the structure of IP ”, Linguistic Inquiry, 20, 3, 365-424. 